Review

\title{
Estimation of the glomerular filtration rate in older individuals with serum creatinine-based equations: A systematic comparison between CKD-EPI and BIS1
}

\author{
Teodoro J. Oscanoa ${ }^{\mathrm{a}, *}$, José P. Amado ${ }^{\mathrm{b}}$, Roman Romero-Ortuno ${ }^{\mathrm{c}}$, José A. Hidalgo ${ }^{\mathrm{d}}$ \\ a Centro de Investigación de Seguridad de Medicamentos de la Facultad de Medicina de la Universidad de San Martín de Porres, Internal Medicine Department, Hospital \\ Almenara ESSALUD, Lima. Av. Grau 800, La Victoria, Lima -13, Peru \\ ${ }^{\mathrm{b}}$ Facultad de Medicina de la Universidad Nacional Mayor de San Marcos, Av Grau 755, Distrito de Lima 15001, Lima, Peru \\ ${ }^{\mathrm{c}}$ Clinical Gerontology Unit, Department of Public Health and Primary Care, University of Cambridge, United Kingdom \\ d Internal Medicine Department, Hospital Almenara ESSALUD, Lima. Av. Grau 800, La Victoria, Lima - 13, Peru
}

\section{A R T I C L E I N F O}

\section{Keywords:}

CKD-EPIcr (Chronic Kidney Disease

Epidemiology Collaboration)

BIS1 (Berlin Initiative Study 1)

Elderly

Glomerular filtration rate

Comparative study

\begin{abstract}
A B S T R A C T
Aim: CKD-EPIcr (Chronic Kidney Disease Epidemiology Collaboration creatinine) and BIS1 (Berlin Initiative Study) are two serum creatinine-based formulae for the estimation of glomerular filtration rate (GFR). However, their comparative accuracy in older people has not been well established. Our aim was to conduct a systematic comparative study of the accuracy of estimation of GFR in older people with these two formulae.

Methods: We conducted a systematic search in Pubmed, EMBASE and Central databases on the validity of the CKD-EPIcr and BIS1 formulae in people aged 60 or more years. The search ranged from 2009 and 2012 for CKDEPIcr and BIS1, respectively, until May 2017. The validity criterion for comparing the formulae was to have a P30 accuracy level equal to or greater than $80 \%$.

Results: Of 1295 identified studies, 16 met our inclusion criteria. Out of 16 studies reporting the accuracy of the CKD-EPIcr formula, only 5 (31.3\%) had P30 scores $\geq 80 \%$ (mean P30 was $77.1 \pm 7.711$ range 55.5-91.7), and out of 9 studies on accuracy using the BIS1 formula, $6(66.7 \%$ ) were $\geq 80 \%$ (mean P30 was $83.88 \pm 9.37$, range 67.0-95.8).

Conclusion: Our results suggest that for the estimation of the GFR in older people, BIS1 formula may be more accurate than CKD-EPIcr.
\end{abstract}

\section{Introduction}

The evaluation of renal function in older people is important for three main reasons. Firstly, the aging process is associated with a gradual impairment of the glomerular filtration rate (GFR), which has been estimated to range between 0.4 and $2.6 \mathrm{ml} / \mathrm{min}$ per year (Bolignano, Mattace-Raso, Sijbrands, \& Zoccali, 2014). There may be significant variation in this average rate of decline; for example, in two large cohort studies (Jiang et al., 2012; Lindeman, Tobin, \& Shock, 1985) it was reported that in 36\% (Baltimore Longitudinal Study of Aging, BLSA) and $44 \%$ of the individuals studied, renal function was not modified during the follow-up period (14 and 5 years, respectively). Secondly, pharmacological therapeutic interventions require knowledge of the current GFR to calculate drug doses, especially those that are eliminated by the kidneys. Thirdly, estimated GFR is the main criterion for the diagnosis of chronic renal failure, which is an independent cardiovascular risk factor, and is associated with an increased risk of mortality, cardiovascular events and hospitalizations (Go, Chertow, Fan, McCulloch, \& Hsu, 2004).

Renal function is assessed with the GFR, which is defined as the volume of purified plasma of an ideal substance (filtered at the renal level and not absorbed or secreted by the renal tubules) per unit of time (expressed in milliliters/minute) (Traynor, Mactier, Geddes, \& Fox, 2006). GFR can be measured and estimated. Measurement of the GFR is performed using exogenous markers (e.g. inulin, iohexol) (Soveri et al., 2014). The estimation of the GFR is performed using endogenous markers of the organism such as creatinine and cystatin C. Available methods of estimation of GFR include: serum creatinine, creatinine clearance, cystatin C and predictive GFR formulae (e.g. Cockcroft and Gault, Modification of Diet in Renal Disease: MDRD).

GFR estimation formulae are frequently used in older people. In 2010 a systematic study evaluated the accuracy of 16 methods of

\footnotetext{
* Corresponding author.

E-mail addresses: toscanoae@usmp.pe (T.J. Oscanoa), jpamadot@gmail.com (J.P. Amado), roman.romero-ortuno@nhs.net (R. Romero-Ortuno), pepinhidalgo@yahoo.com (J.A. Hidalgo).
} 
Box 1

Creatinine-based equations to predict glomerular filtration rate in older patients.

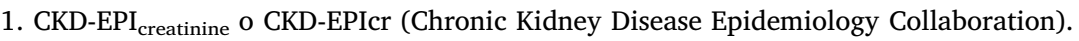

\begin{tabular}{lll}
\hline & Serum creatinine $(\mathrm{mg} / \mathrm{dl})$ & Equation \\
\hline female & $<0.7$ & $144 \mathrm{x}(\mathrm{Scr} / 0.7)^{-0.329} \times 0.993^{\text {age }}(\times 1.159$, if black $)$ \\
\multirow{2}{*}{ male } & $>0.7$ & $144 \mathrm{x}(\mathrm{Scr} / 0.7)^{-1.209} \times 0.993^{\text {age }}(\times 1.159$, if black $)$ \\
& $<0.9$ & $141 \times(\mathrm{Scr} / 0.9)^{0.411} \times 0.993^{\text {age }}(\times 1.159$, if black $)$ \\
& $>0.9$ & $141 \times(\mathrm{Scr} / 0.9)^{-1.209} 0.993^{\text {age }}(\times 1.159$, if black $)$
\end{tabular}

Reference: Levey AS, et al. A new equation to estimate glomerular filtration rate. Ann Intern Med. 2009;150(9): 604-612.

2. BIS1 (Berlin Initiative Study 1).

BIS1 $=3736 \times$ creatinine $^{-0.87} \times$ age $^{-0.95} \times 0.82$ (if female)

Reference: Schaeffner ES, et al. Two novel equations to estimate kidney function in persons aged 70 years or older.Ann Intern Med. 2012

Oct 2;157(7):471-81

measuring GFR, including 14 formulae. Of all methods included in the study, the measurement of the serum concentration of cystatin C, Cockcroft and Gault formula and MDRD, seemed the most accurate (Van Pottelbergh, Van Heden, Matheï, \& Degryse, 2010). The Cockcroft and Gault formula, created in 1976, is actually an estimate of creatinine clearance and not of GFR and does not appear to be accurate enough in people with chronic kidney disease. Recently, new formulae have been published with their respective validations in older people such as CKDEPI (Chronic Kidney Disease Epidemiology Collaboration) $(8,9)$ and BIS (Berlin Initiative Study) (Schaeffner et al., 2012a). The full details of the latter two formulae are shown in Box 1.

The CKD-EPI formula was developed by Levey et al. of Tufts Medical Center, Boston, USA, based on the analysis of a cohort of 2019 patients aged $\geq 70$ years (Levey et al., 2009). Two studies (Levey et al., 2009; Michels et al., 2010) have noted that the CKD-EPIcr formula is as accurate as MDRD when the GFR is $<60 \mathrm{ml} / \mathrm{min} / 1.73 \mathrm{~m}^{2}$, but its accuracy is greater than MDRD if the GFR is $>60 \mathrm{ml} / \mathrm{min} / 1.73 \mathrm{~m}^{2}$. There are two variations of this formula. One is based on cystatin alone (CKDEPIcys), and the second uses both creatinine and cystatin (CKD- EPIcrcys). The inclusion of cystatin $\mathrm{C}$ aims to improve the variability of creatinine in older people due to sarcopenia, which may be associated with aging. A recent study favored CKD-EPIcr-cys as showing greater precision and accuracy than formulae containing Cystatin C or creatinine only (Inker et al., 2012). In 2012, Elke S. Schaeffner and colleagues published two formulae that emerged from studies in white people aged $>70$ years (Schaeffner et al., 2012a). These formulae were validated in two studies (Koppe, Klich, Dubourg, Ecochard, \& Hadj-Aissa, 2013a; Liu, Chen, \& Wang, 2013a). The first formula (BIS1) is based on serum creatinine, whereas the second (BIS2) uses both serum creatinine and cystatin C. In a comparative study between MDRD, CKD-EPI and BIS1 formulae for estimation of GFR, BIS1 was shown to be the most reliable in older people, especially in those with stage 1 and stage 2 chronic kidney disease (Liu et al., 2013a). A recent systematic study on the validity of the MDRD formula in older people found that, out of 9 studies reviewed, only one met the criterion of acceptable validity in older people (P30 greater than 80\%). Therefore, the authors concluded that this formula may not be valid in this population group (Eppenga et al., 2015).

CKD-EPIcr and BIS1 are two serum creatinine-based formulae for the estimation of GFR. However, their comparative accuracy in older people has not been well established. Our aim was to conduct a systematic comparative study of the accuracy of estimation of GFR in older people with these two formulae.

\section{Methods}

\subsection{Search strategy}

We conducted a systematic search in MEDLINE, EMBASE, and Central databases of published studies on the validity of the CKD-EPIcr and BIS1 formulae in population aged 60 or more years of age. The search covered the period between 2009 and 2012 for CKD-EPIcr and BIS1, respectively (the dates are related to the year of publication of the formulae), until May 31, 2017. In addition, other databases (Google Scholar, Database of Abstracts of Systematic Reviews from the Cochrane Library) and a secondary search based on reference lists of original articles were used. The terms used for the overall search strategy are listed in File S1 in Appendix A. Titles and abstracts were reviewed independently by two authors (JA and JH). Disagreements between two reviewers were resolved by consensus.

\subsection{Selection criteria}

The inclusion criteria for the studies were: a) study population aged 60 years or older, or where other age groups were included, specific data for the $60+$ group were available; b) the estimation of the GFR made against a reference method such as Inulin, ${ }^{51} \mathrm{Cr}$-EDTA (chromium 51 labeled with ethylenediaminetetraacetic acid), 99Tc-DTPA (technetium 99 labeled with diethylene triamine pentacetic acid), iohexol, or iothalamate (Brandstrom, Grzegorczyk, \& Jacobsson, 1998; James, Lewis, \& Tan, 2007; O'Reilly et al., 1986); c) results included statistical analysis of bias, precision and accuracy. We excluded studies that did not have results of accuracy (P30), case reports, abstracts and posters, as well as studies with data already used in previous publications.

Three instruments were used to evaluate the formulae: bias, precision and accuracy. The bias and precision were used as a representation of systematic error and random error, respectively. Accuracy was used as a marker of both bias and precision. Bias is defined as the mean (or median) difference between the estimated GFR (eGFR) and measured GFR (mGFR) (Delanaye, Pottel, \& Botev, 2013). Precision was defined operationally as standard deviation (SD) or interquartile range (IQR) of the bias, and represents the spread of $68 \%$ (assuming normal distribution) or $50 \%$ of the values around the bias, respectively (Delanaye et al., 2013). Accuracy is the percentage of eGFRs within a defined range of their respective mGFRs; in other words, it is a percentage of the eGFR within $\mathrm{k} \%$ of the mGFR (Pk\%). P30 is the proportion of values of eGFR that lie within $30 \%$ of mGFR. For example, if P30 (which represents the number that is the 30 th percentile) is $50 \%$, it means that in half of the cases the eGFR falls within $\pm 30 \%$ of the mGFR (Delanaye et al., 2013). 
In serum creatinine-based equations in older people, it is difficult to achieve a $100 \%$ of P30 due to different factors affecting the results of serum creatinine measurement (e.g. analytical variation of the measurement of serum creatinine levels and decreased muscle mass associated with aging or sarcopenia) (Delanaye et al., 2013). Currently, it is considered that a P30 of $80 \%$ or higher is an indicator of sufficient validity (Eppenga et al., 2015). In order to compare the two studied formulae (CKD-EPIcr and BIS1), a validity indicator was considered when accuracy expressed in P30 was $\geq 80 \%$.

The quality of the studies was assessed by using the guidelines of the updated Quality Assessment of Diagnostic Accuracy Studies (QUADAS2) tool (Whiting et al., 2011). The systematic review was undertaken according to the Preferred Reporting Items for Systematic Reviews and Meta-Analyses (PRISMA) checklist (Liberati et al., 2009).

\section{Results}

We initially identified 1295 abstracts with the search terms listed in File S1 in Appendix A. In total, we included 16 studies (Bevc, Hojs, Ekart, Gorenjak, \& Puklavec, 2011; Buron et al., 2011; Changjie et al., 2017; David-Neto et al., 2016; Fan et al., 2015; Kilbride et al., 2013; Koppe, Klich, Dubourg, Ecochard, \& Hadj-Aissa, 2013b; Lopes et al., 2013; Liu et al., 2013b; Nyman et al., 2014; Schaeffner et al., 2012b; Segarra et al., 2011; Vidal-Petiot et al., 2014; Werner et al., 2017; Ye et al., 2014; Zhu et al., 2014). Fig. 1 shows the flow diagram of selected studies. The most relevant information from the selected studies included in the review is presented in Table 1 . The quality assessment of all included studies based on QUADAS-2 is show in Table 2.

\subsection{Study characteristics}

The 16 studies included were conducted in 9 different countries (China: 4; France: 3; Sweden: 2; Brazil: 2; Slovenia: 1; Spain: 1; United Kingdom: 1; Iceland: 1; Germany: 1). The mean size of the total population studied was 572 (range: 95-2017). The age range was 60 to 97 years old. Seven studies included people $<70$ years of age in the total analysis.

The reference method for the measurement of GFR was iohexol (7

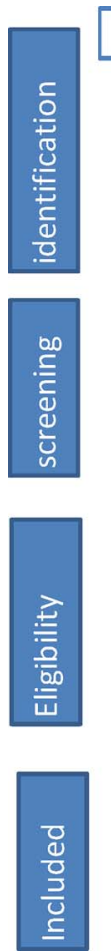

Flow diagram of the selection of eligible studies
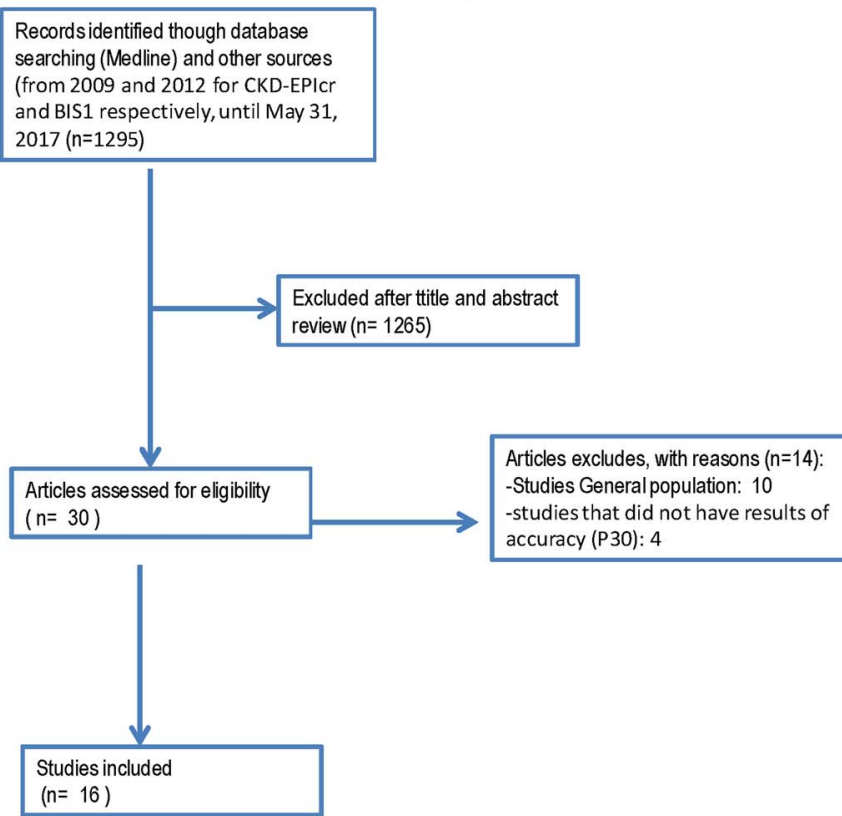

studies), 99mTc-DTPA (4 studies), 51Cr-EDTA (3 studies) and inulin (2 studies). Recruitment included participants from the community (5 studies), patients referred for measurement of GFR (4), hospitalized patients (5) and kidney transplant recipients (2). Serum creatinine determination was performed using the enzymatic method in 13 studies, mostly calibrated with the IDMS (Isotope Dilution Mass Spectrometry) method. Jaffé method was used in 3 studies (Table 1).

\subsection{Performance of CKD-EPICr and BIS1 equations in estimating GFR in older people}

Out of 16 studies on the accuracy of CKD-EPIcr using P30 as indicator, $5(31.3 \%)$ were $\geq 80 \%$. The P30 median was 77.0 (range 55.5-91.7). Using the same indicator, of a total of 9 studies on BIS1 accuracy, $6(66.6 \%$ ) had P30 $\geq 80 \%$. The P30 median was 83.3 (range 67.0-95.8).

\subsection{Subgroup analyses}

We analyzed the effect of age on the accuracy of the formulae. When the population group was older than 70 years (mean age $>70$ ), of the 7 studies performed with BIS1, 6 studies $(85.7 \%)$ had a P30 $\geq 80 \%$. In this population group, 11 studies on CKD-EPIcr were performed, of which only $4(36.4 \%)$ studies had a P30 $\geq 80 \%$ (Table 1 ).

When the effect of the GFR on the accuracy of the formulae was studied, only 8 of the 16 studies described their findings in detail, although the GFR intervals were not uniform (Table 3). When GFR $\geq 60 \mathrm{ml} / \mathrm{min} / 1.73 \mathrm{~m}^{2}$, with the BIS1 formula 5 of 5 studies (100\%) reported a P30 $\geq 80 \%$; with the CKD-EPIcr formula 5 out of 7 studies (71.4\%) had a $\mathrm{P} 30 \geq 80 \%$. In one study, it was not possible to compare because the cutoff point was GFR $>45 \mathrm{ml} / \mathrm{min} / 1.73 \mathrm{~m}^{2}$ (in the latter study, both BIS1 and CKD-EPIcr had a P30 $\geq 80 \%$ (Werner et al., 2017). Comparisons in other GFR ranges (e.g. $<60$ or $>45$ ) were not possible due to the very low number of studies (see Table 3 ).

Of the 16 included studies, 9 compared the two formulae in the same study. Among these studies, 6 achieved a P30 > 80\% with BIS1 and only 3 with CKD-EPIcr.
Fig. 1. Flow diagram of the selection of eligible studies. 


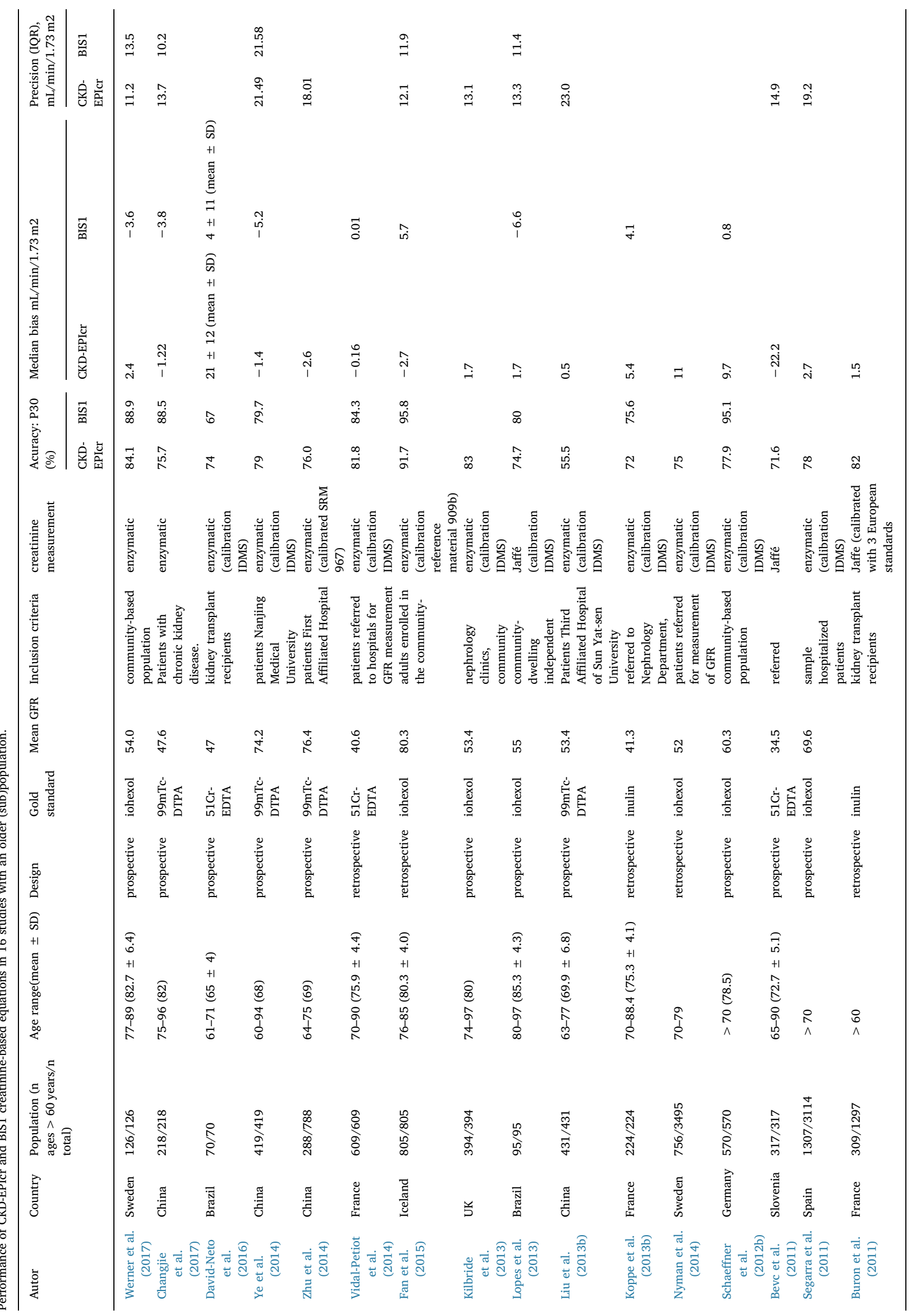


Table 2

Quality assessment of individual studies. Quality Assessment of Diagnostic Accuracy Studies (QUADAS-2) tool.

\begin{tabular}{|c|c|c|c|c|c|c|c|}
\hline \multirow[t]{2}{*}{ Study } & \multicolumn{4}{|l|}{ Risk of Bias } & \multicolumn{3}{|c|}{ Applicability Concerns } \\
\hline & Patient Selection & Index Test & Reference Standard & Flow and Timing & Patient Selection & Index Test & Reference Standard \\
\hline Werner et al. (2017) & $\mathrm{L}$ & $\mathrm{L}$ & $\mathrm{L}$ & $\mathrm{L}$ & $\mathrm{L}$ & $\mathrm{L}$ & $\mathrm{L}$ \\
\hline Changjie et al. (2017) & $\mathrm{L}$ & $\mathrm{L}$ & $\mathrm{L}$ & $\mathrm{L}$ & $\mathrm{L}$ & $\mathrm{L}$ & $\mathrm{L}$ \\
\hline David-Neto et al. (2016) & $\mathrm{L}$ & $\mathrm{L}$ & $\mathrm{L}$ & $\mathrm{L}$ & $\mathrm{L}$ & $\mathrm{L}$ & $\mathrm{L}$ \\
\hline Ye et al. (2014) & $\mathrm{H}$ & $\mathrm{L}$ & $\mathrm{L}$ & $\mathrm{L}$ & $\mathrm{L}$ & $\mathrm{L}$ & $\mathrm{L}$ \\
\hline Zhu et al. (2014) & $\mathrm{H}$ & $\mathrm{L}$ & $\mathrm{L}$ & $\mathrm{L}$ & $\mathrm{L}$ & $\mathrm{L}$ & $\mathrm{L}$ \\
\hline Vidal-Petiot et al. (2014) & $\mathrm{U}$ & $\mathrm{L}$ & $\mathrm{L}$ & $\mathrm{L}$ & $\mathrm{L}$ & $\mathrm{L}$ & $\mathrm{L}$ \\
\hline Fan et al. (2015) & $\mathrm{L}$ & $\mathrm{L}$ & $\mathrm{L}$ & $\mathrm{L}$ & $\mathrm{L}$ & $\mathrm{L}$ & $\mathrm{L}$ \\
\hline Kilbride et al. (2013) & $\mathrm{L}$ & $\mathrm{L}$ & $\mathrm{L}$ & $\mathrm{L}$ & $\mathrm{L}$ & $\mathrm{L}$ & $\mathrm{L}$ \\
\hline Lopes et al. (2013) & $\mathrm{L}$ & $\mathrm{L}$ & $\mathrm{L}$ & $\mathrm{H}$ & $\mathrm{L}$ & $\mathrm{L}$ & $\mathrm{L}$ \\
\hline Liu et al. (2013b) & $\mathrm{H}$ & $\mathrm{L}$ & $\mathrm{L}$ & $\mathrm{L}$ & $\mathrm{L}$ & $\mathrm{L}$ & $\mathrm{L}$ \\
\hline Koppe et al. (2013b) & $\mathrm{L}$ & $\mathrm{L}$ & $\mathrm{L}$ & $\mathrm{H}$ & $\mathrm{L}$ & $\mathrm{L}$ & $\mathrm{L}$ \\
\hline Nyman et al. (2014) & $\mathrm{L}$ & $\mathrm{L}$ & $\mathrm{L}$ & $\mathrm{L}$ & $\mathrm{L}$ & $\mathrm{L}$ & $\mathrm{L}$ \\
\hline Schaeffner et al. (2012b) & $\mathrm{L}$ & $\mathrm{L}$ & $\mathrm{L}$ & $\mathrm{L}$ & $\mathrm{L}$ & $\mathrm{L}$ & $\mathrm{L}$ \\
\hline Bevc et al. (2011) & $\mathrm{L}$ & $\mathrm{L}$ & $\mathrm{L}$ & $\mathrm{L}$ & $\mathrm{L}$ & $\mathrm{L}$ & $\mathrm{L}$ \\
\hline Segarra et al. (2011) & $\mathrm{L}$ & $\mathrm{L}$ & $\mathrm{L}$ & $\mathrm{L}$ & $\mathrm{L}$ & $\mathrm{L}$ & $\mathrm{L}$ \\
\hline Buron et al. (2011) & $\mathrm{L}$ & $\mathrm{L}$ & $\mathrm{L}$ & $\mathrm{H}$ & $\mathrm{L}$ & $\mathrm{L}$ & $\mathrm{L}$ \\
\hline
\end{tabular}

$\mathrm{L}=$ low risk, $\mathrm{H}=$ High risk; $\mathrm{U}=$ unclear risk

\section{Discussion}

Results in the present study suggest that in older people BIS1 may estimate GFR more accurately than CKD-EPIcr, when using the indicator P30 $\geq 80 \%$, especially in individuals aged $>70$ years. The accuracy of BIS1 may be higher than CKD-EPIcr especially in patients with GFR $\geq 60 \mathrm{ml} / \mathrm{min} / 1.73 \mathrm{~m}^{2}$.

Possible explanations for our findings may be in relation to the characteristics of the investigated formulae, samples, markers for measuring clearance, methods of measuring GFR (plasma and urine clearance) and calibration of serum creatinine (Koppe et al., 2013b). The BIS1 formula was developed and validated from a sample of people older than 70 years, whereas CKD-EPIcr only included a small percentage of older people (Levey et al., 2009). BIS1 validation studies used uniform and accurate reference methods for mGFR (iohexol clearance), while CKD-EPIcr used different methods (plasma clearance of iohexol, urinary clearance of $99 \mathrm{mTc}$-DTPA, renal clearance of nonradiolabeled iothalamate, 51Cr-EDTA or inulin) (Koppe et al., 2013b). Validation of BIS1 was performed with isotope dilution mass spectrometry, while in CKD-EPIcr the lack of standardization of the creatinine assay method was observed in some studies. On the other hand, in the present study none of the two formulae investigated had a P30 $\geq 80 \%$ when GFR $<30 \mathrm{ml} / \mathrm{min} / 1.73 \mathrm{~m}^{2}$, probably because the two formulae come from studies with populations with normal GFR or $>30 \mathrm{ml} / \mathrm{min} / 1.73 \mathrm{~m}^{2}$.

The evolution of the accuracy evaluation of creatinine-based GFR estimation formulae for older people has been relatively rapid in the last decade. Ten years ago, the classic Cockcroft-Gault formula was found to be less accurate than the MDRD (P30 of 69\% versus $83 \%$ respectively) (Levey, Inker, \& Coresh, 2014; Stevens, Coresh, \& Feldman, 2007). In the year 2012, a systematic review was published, in which it was found that the CKD-EPIcr formula is more accurate than the MDRD (Earley, Miskulin, Lamb, Levey, \& Uhlig, 2012). These findings may have caused an increase in its use since 2014 especially in Australia, France and some laboratories in the USA (Earley et al., 2012; Levey et al., 2014; Stevens et al., 2007). A recent study found that the BIS-1 formula is better than the CKD-EPIcr formula for risk stratification of chronic kidney disease in older people with cardiovascular disease (Tarantini et al., 2016). If new studies confirm the findings of the present study, the frequency of use of the BIS1 formula for the estimation of GFR in people older than 70 years is likely to increase.

When considering the accuracy and cost-effectiveness of GFR estimation, it is important to compare formulae based on creatinine versus those based on cystatin alone or associated with creatinine. In this case, it is necessary to take into account three facts. Firstly, the applicability of the determination of cystatin $\mathrm{C}$ in routine clinical practice is low, due to its high cost (Creatinine: \$ 0.25 versus cystatin C: about \$3.00) (Filler et al., 2005). Secondly, a recent meta-analytic study with 16 cohorts reported that both CKD-EPIDs with cystatin and those combined with creatinine are better predictors of cardiovascular mortality than the CKD-EPI formula with creatinine alone. However, the study population included younger participants, resulting in a heterogeneous muscle mass. Therefore, the findings may not applicable to older people (Shlipak, Matsushita, Arnlov, Inker, \& Katz, 2013). Thirdly, in a recent prospective study of a cohort of 1165 women over 70 years of age, CKDEPI with cystatin alone or creatinine-associated formulae did not improve the predictive ability for clinical events (i.e. mortality and hospitalization for cardiovascular events) compared to CKD-EPIcr (Lim et al., 2014).

Our study has limitations. We have included studies that used P30 as an indicator of accuracy, and therefore, the exclusion of studies with other forms of expression of results (e.g. mean square error) could vary the comparison between the formulae studied. The number of studies included with BIS1 is relatively small, which implies that we should be attentive to the results of other studies in the future to confirm present findings. On the other hand, when GFR $<30$ none of the two formulae had a P30 $\geq 80 \%$, although these findings were described in only 4 studies (Changjie et al., 2017; Koppe et al., 2013b; Liu et al., 2013b; Vidal-Petiot et al., 2014). The effectiveness of formulae to correctly classify patients with chronic renal failure as compared to the reference test has not been studied, nor the effectiveness of formulae to predict outcomes such as mortality. These last two limitations require more studies with longer follow-up periods. BIS1 was only proposed in 2012.

In summary, this systematic review showed that for the estimation of the GFR in older people, the BIS1 formula may be more accurate than CKD-EPIcr. 
Appendix A

File S1: Search terms

Data base: Pubmed (MEDLINE). < Database inception January 2009-May $2017>$

Search terms:

(a) "Glomerular Filtration Rate"[All Fields] AND ("Predictive Value of Tests"[Mesh] OR "Reference Values"[Mesh]).

(b) Pubmed: Chronic AND Kidney AND Disease AND Epidemiology AND Collaboration

(c) Pubmed: Berlin AND Initiative AND Study.

Pubmed: (a) AND ((b) OR (c))

Data base: EMBASE. < Database inception January 2009-May $2017>60+$ years.

Search terms:

("Glomerular Filtration Rate" AND ("Predictive Value of Tests" OR "Reference Values")) AND ((Chronic AND Kidney AND Disease AND Epidemiology AND Collaboration) OR (Berlin AND Initiative AND Study)).

\section{References}

Bevc, S., Hojs, R., Ekart, R., Gorenjak, M., \& Puklavec, L. (2011). Simple cystatin C formula compared to sophisticated CKD-EPI formulas for estimation of glomerular filtration rate in the elderly. Therapeutic Apheresis and Dialysis, 15(3), 261-268.

Bolignano, D., Mattace-Raso, F., Sijbrands, E. J., \& Zoccali, C. (2014). The aging kidney revisited: A systematic review. Ageing Research Reviews, 14, 65-80.

Brandstrom, E., Grzegorczyk, A., Jacobsson, L., et al. (1998). GFR measurement with iohexol and 51Cr-EDTA. A comparison of the two favoured GFR markers in Europe. Nephrology Dialysis Transplantation, 13, 1176-1182.

Buron, F., Hadj-Aissa, A., Dubourg, L., Morelon, E., Steghens, J. P., Ducher, M., et al. (2011). Estimating glomerular filtration rate in kidney transplant recipients: Performance over time of four creatinine-based formulas. Transplantation, 92(9), 1005-1011.

Changjie, G., Xusheng, Z., Feng, H., Shuguang, Q., Jianwen, L., \& Junzhou, F. (2017). Evaluation of glomerular filtration rate by different equations in Chinese elderly with chronic kidney disease. International Urology and Nephrology, 49(1), 133-141.

David-Neto, E., Kamada Triboni, A. H., Ramos, F., Agena, F., Zocoler Galante, N., Altona, M., et al. (2016). Evaluation of MDRD4, CKD-EPI, BIS-1, and modified Cockcroft-Gault equations to estimate glomerular filtration rate in the elderly renal-transplanted recipients. Clinical Transplantation, 30(12), 1558-1563.

Delanaye, P., Pottel, H., \& Botev, R. (2013). Con: Should we abandon the use of the MDRD equation in favour of the CKD-EPI equation? Nephrology Dialysis Transplantation, 28(6), 1396-1403.

Earley, A., Miskulin, D., Lamb, E. J., Levey, A. S., \& Uhlig, K. (2012). Estimating equations for glomerular filtration rate in the era of creatinine standardization: A systematic review? Annals of Internal Medicine, 156(11), 785-795.

Eppenga, W. L., Kramers, C., Derijks, H. J., Wensing, M., Wetzels, J. F., \& De Smet, P. A. (2015). Individualizing pharmacotherapy in patients with renal impairment: The validity of the modification of diet in renal disease formula in specific patient populations with a glomerular filtration rate below $60 \mathrm{ml} / \mathrm{min}$. A systematic review. PUBLIC LIBRARY OF SCIENCE, 10(3), e0116403.

Fan, L., Levey, A. S., Gudnason, V., Eiriksdottir, G., Andresdottir, M. B., Gudmundsdottir, H., et al. (2015). Comparing GFR estimating equations using cystatin C and creatinine in elderly individuals. Journal of the American Society of Nephrology, 26(8), 1982-1989.

Filler, G., Bökenkamp, A., Hofmann, W., Le Bricon, T., Martínez-Brú, C., \& Grubb, A. (2005). Cystatin C as a marker of GFR-history, indications, and future research. Clinical Biochemistry, 38(1), 1-8.

Go, A. S., Chertow, G. M., Fan, D., McCulloch, C. E., Hsu, C. Y., et al. (2004). Chronic kidney disease and the risks of death, cardiovascular events, and hospitalization. The New England Journal of Medicine, 351, 1296-1305.

Inker, L. A., Schmid, C. H., Tighiouart, H., Eckfeldt, J. H., Feldman, H. I., Greene, T., et al. (2012). Estimating glomerular filtration rate from serum creatinine and cystatin C. The New England Journal of Medicine, 367(1), 20-29.

James, T. J., Lewis, A. V., Tan, G. D., et al. (2007). Validity of simplified protocols to estimate glomerular filtration rate using iohexol clearance. Annals of Clinical Biochemistry, 44, 369-376.

Jiang, S., Sun, X., Gu, H., Chen, Y., Xi, C., Qiao, X., et al. (2012). Age-related change in kidney function, its influencing factors, and association with asymptomatic carotid atherosclerosis in healthy individuals-a 5-year follow-up study. Maturitas, 73, 230-238.

Kilbride, H. S., Stevens, P. E., Eaglestone, G., Knight, S., Carter, J. L., Delaney, M. P., et al. (2013). Accuracy of the MDRD (modification of diet in renal disease) study and CKDEPI (CKD epidemiology collaboration) equations for estimation of GFR in the elderly. American Journal of Kidney Diseases, 61(1), 57-66. 
Koppe, L., Klich, A., Dubourg, L., Ecochard, R., \& Hadj-Aissa, A. (2013a). Performance of creatinine-based equations compared in older patients. Journal of Nephrology, 26(4), 716-723.

Koppe, L., Klich, A., Dubourg, L., Ecochard, R., \& Hadj-Aissa, A. (2013b). Performance of creatinine-based equations compared in older patients. Journal of Nephrology, 26(4), 716-723.

Levey, A. S., Stevens, L. A., Schmid, C. H., Zhang, Y. L., Castro, A. F., 3rd, Feldman, H. I., et al. (2009). A new equation to estimate glomerular filtration rate. Annals of Internal Medicine, 150, 604-612.

Levey, A. S., Inker, L. A., \& Coresh, J. (2014). GFR estimation: From physiology to public health. American Journal of Kidney Diseases, 63(5), 820-834.

Liberati, A., Altman, D. G., Tetzlaff, J., Mulrow, C., Gøtzsche, P. C., Ioannidis, J. P., et al. (2009). The PRISMA statement for reporting systematic reviews and meta-analyses of studies that evaluate health care interventions: Explanation and elaboration. Journal of Clinical Epidemiology, 62(10), e1-34.

Lim, W. H., Lewis, J. R., Wong, G., Turner, R. M., Lim, E. M., Thompson, P. L., et al. (2014). Comparison of estimated glomerular filtration rate by the chronic kidney disease epidemiology collaboration (CKD-EPI) equations with and without Cystatin C for predicting clinical outcomes in elderly women. PUBLIC LIBRARY OF SCIENCE, 9(9), e106734.

Lindeman, R. D., Tobin, J., \& Shock, N. W. (1985). Longitudinal studies on the rate of decline in renal function with age. Journal of the American Geriatrics Society, 33, $278-285$.

Liu, X., Chen, J., Wang, C., et al. (2013a). Assessment of glomerular filtration rate in elderly patients with chronic kidney disease. International Urology and Nephrology, 45(5), 1475-1482.

Liu, X., Ma, H., Huang, H., Wang, C., Tang, H., Li, M., et al. (2013b). Is the chronic kidney disease epidemiology collaboration creatinine-cystatin $\mathrm{C}$ equation useful for glomerular filtration rate estimation in the elderly? Clinical Interventions in Aging, 8, $1387-1391$

Lopes, M. B., Araújo, L. Q., Passos, M. T., Nishida, S. K., Kirsztajn, G. M., Cendoroglo, M. S., et al. (2013). Estimation of glomerular filtration rate from serum creatinine and cystatin C in octogenarians and nonagenarians. BMC Nephrology, 14, 265. http://dx. doi.org/10.1186/1471-2369-14-265.

Michels, W. M., Grootendorst, D. C., Verduijn, M., Elliott, E. G., Dekker, F. W., \& Krediet, R. T. (2010). Performance of the Cockcroft-Gault, MDRD, and new CKD-EPI formulas in relation to GFR, age, and body size. Clinical Journal of the American Society of Nephrology, 5(6), 1003-1009.

Nyman, U., Grubb, A., Larsson, A., Hansson, L. O., Flodin, M., Nordin, G., et al. (2014). The revised Lund-Malmö GFR estimating equation outperforms MDRD and CKD-EPI across GFR, age and BMI intervals in a large Swedish population. Clinical Chemistry and Laboratory Medicine, 52(6), 815-824.

O'Reilly, P. H., Brooman, P. J., Martin, P. J., Pollard, A. J., Farah, N. B., \& Mason, G. C. (1986). Accuracy and reproducibility of a new contrast clearance method for the determination of glomerular filtration rate? British Medical Journal (Clinical Research
Edition), 293(6541), 234-236

Schaeffner, E. S., Ebert, N., Delanaye, P., Frei, U., Gaedeke, J., Jakob, O., et al. (2012a). Two novel equations to estimate kidney function in persons aged 70 years or older. Annals of Internal Medicine, 157(7), 471-481.

Schaeffner, E. S., Ebert, N., Delanaye, P., Frei, U., Gaedeke, J., Jakob, O., et al. (2012b). Two novel equations to estimate kidney function in persons aged 70 years or older? Annals of Internal Medicine, 157(7), 471-481.

Segarra, A., de la Torre, J., Ramos, N., Quiroz, A., Garjau, M., Torres, I., et al. (2011). Assessing glomerular filtration rate in hospitalized patients: A comparison between CKD-EPI and four cystatin C-based equations. Clinical Journal of the American Society of Nephrology, 6(10), 2411-2420.

Shlipak, M. G., Matsushita, K., Arnlov, J., Inker, L. A., Katz, R., et al. (2013). Cystatin C versus creatinine in determining risk based on kidney function. The New England Journal of Medicine, 369, 932-943.

Soveri, I., Berg, U. B., Björk, J., Elinder, C. G., Grubb, A., Mejare, I., et al. (2014) Measuring GFR: A systematic review. American Journal of Kidney Diseases, 64(3), 411-424.

Stevens, L. A., Coresh, J., Feldman, H. I., et al. (2007). Evaluation of the modification of diet in renal disease study equation in a large diverse population? Journal of the American Society of Nephrology, 18(10), 2749-2757.

Tarantini, L., McAlister, F. A., Barbati, G., Ezekowitz, J. A., Cioffi, G., Faggiano, P., et al (2016). Chronic kidney disease and prognosis in elderly patients with cardiovascular disease: Comparison between CKD-EPI and Berlin Initiative Study-1 formulas. European Journal of Preventive Cardiology, 23(14), 1504-1513.

Traynor, J., Mactier, R., Geddes, C. C., \& Fox, J. G. (2006). How to measure renal function in clinical practice. BMJ: British Medical Journal, 333(7571), 733.

Van Pottelbergh, G., Van Heden, L., Matheï, C., \& Degryse, J. (2010). Methods to evaluate renal function in elderly patients: A systematic literature review. Age and Ageing, 39(5), 542-548.

Vidal-Petiot, E., Haymann, J. P., Letavernier, E., Serrano, F., Clerici, C., Boffa, J. J., et al. (2014). External validation of the BIS (Berlin Initiative Study)-1 GFR estimating equation in the elderly. American Journal of Kidney Diseases, 63(5), 865-867.

Werner, K., Pihlsgård, M., Elmståhl, S., Legrand, H., Nyman, U., \& Christensson, A. (2017). Combining cystatin $C$ and creatinine yields a reliable glomerular filtration rate estimation in older adults in contrast to $\beta$-trace protein and $\beta 2$-microglobulin. Nephron. http://dx.doi.org/10.1159/000473703.

Whiting, P. F., Rutjes, A. W., Westwood, M. E., Mallett, S., Deeks, J. J., Reitsma, J. B., et al. (2011). QUADAS-2: A revised tool for the quality assessment of diagnostic accuracy studies. Annals of Internal Medicine, 155(8), 529-536.

Ye, X., Wei, L., Pei, X., Zhu, B., Wu, J., \& Zhao, W. (2014). Application of creatinine- and/ or cystatin C-based glomerular filtration rate estimation equations in elderly Chinese. Clinical Interventions in Aging, 9, 1539-1549.

Zhu, Y., Ye, X., Zhu, B., Pei, X., Wei, L., Wu, J., et al. (2014). Comparisons between the 2012 new CKD-EPI (chronic kidney disease epidemiology collaboration) equation and other four approved equations. PUBLIC LIBRARY OF SCIENCE, 9(1), e84688. 